\title{
CIÊNCIA E INOVAÇÃO
}

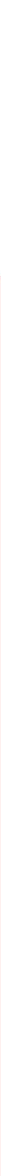


Sob a pressão seletiva das necessidades, a espécie humana aprendeu a pesquisar, acumular conhecimento e inovar. Essa foi a estratégia central na evolução humana, que tem a ciência e a inovação como faces complementares da mesma moeda. No Brasil, superada a fase predatória colonial, essa estratégia de acumulação de conhecimento como premissa da inovação se faz sentir após a Segunda Guerra Mundial, com os investimentos em ciência e tecnologia para garantir a soberania e o desenvolvimento econômico e social. O modelo adotado foi o de se criar um forte programa de capacitação no país e no exterior juntamente com um programa de apoio à pesquisa científica, promovendo-se numa primeira etapa a geração de conhecimento que, fatalmente, levaria à sua utilização na inovação.

Apesar da falta de consenso em relação aos indicadores utilizados para medir e comparar nosso grau de desenvolvimento científico e tecnológico, os resultados sugerem que já alcançamos um estágio respeitável, tanto do ponto de vista qualitativo quanto quantitativo, na geração do conhecimento necessário para darmos um salto em relação à inovação no contexto de uma sociedade moderna que busca o desenvolvimento econômico e social em bases sustentáveis.

Palavras-chave: estratégia de desenvolvimento, conhecimento, ciência, inovação, estratégia.

\section{ABSTRACT}

Necessity exerted a selection pressure on the human species, who learned to research, to accumulate knowledge and to innovate. Such strategy was key to human evolution; and science and innovation are the two complemetary sides of the same coin. In Brazil, after the predatory phase of the colonial rule had ended, such strategy of accumulating knowledge as a premise of innovation can be noticed in the post-Second World War period, when investments were made in science and technology to ensure sovereignty as well as social and economic development. The model adopted then was that of creating a solid training program both in the country and abroad, along with a support program for scientific research. Thus, at their early phase those programs sought to promote the generation of knowledge, which eventually would lead to its use in innovation.

Despite the lack of consensus in relation to the indicators used to measure and compare our level of scientific and technological development, the results indicate that we have achieved a respectable stature - both qualitative and quantitative-wise - of generating knowledge needed for us to take a leap forward in terms of innovation, in a context of a modern society seeking social and economic devopment on sustainable bases.

Keywords: development strategy, knowledge, science, innovation, strategy. 


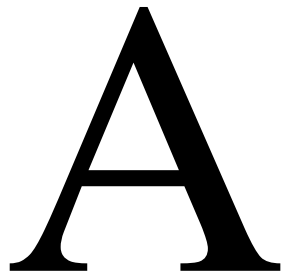

evolução darwiniana das espécies atribuiu grande vantagem competitiva ao desenvolvimento cognitivo alcançado pelos humanos.

As espécies hominoides que, há cerca de 100 mil anos, começaram a se diferenciar de seus ancestrais primatas desenvolveram a peculiar capacidade de compreender seu entorno e elaborar instrumentos e estratégias que lhes permitiram conquistar ampla primazia na biota terrestre em espaço de tempo relativamente pequeno quando comparado à história biológica do planeta. Assim, conhecer e aplicar criativamente o conhecimento adquirido têm sido estratégias centrais na evolução da espécie humana e da sociedade. Um exemplo clássico foi o domínio empírico de técnicas agrícolas e da tecnologia na produção de metais, instrumentais para a forte expansão colonizadora humana que se propagou por todos os continentes do planeta. $\mathrm{O}$ avanço da organização social e a progressiva divisão cooperativa do trabalho contribuíram para o surgimento de indivíduos e grupos dedicados exclusivamente ao desenvolvimento do conhecimento per se, iconizados, por exemplo, nos iniciados do Egito antigo e nas escolas filosóficas gregas, que permitiram o avanço sobre o desconhecido de forma mais rápida e eficiente do que a que até então vinha sendo alcançada pela adição incremental do conhecimento, determinada pelas necessidades imediatas.

Nasce assim a ciência moderna, que, a não ser no interregno obscurantista da Idade Média, passa a ter papel vital para o avanço tecnológico, econômico e social do planeta. $\mathrm{O}$ avanço do conhecimento científico, frequentemente motivado pela curiosidade ou pela necessidade, inexoravelmente promove o desenvolvimento tecnológico com fortes impactos econômicos e sociais.

Tomando como exemplo a história da física, vemos a dramática saga do conhecimento astronômico permitir o desenvolvimento de sistemas de localização e navegação, centrais para a expansão da civilização ociden- tal pelos continentes terrestres nos grandes descobrimentos. A aventura observacional e matemática da mecânica newtoniana enseja a ampla profusão de instrumentos e máquinas que multiplicam a capacidade de produzir força e tração, bem como o desenvolvimento de armamentos balísticos com grande precisão essenciais aos esforços de guerra e dominação tão comuns nos séculos subsequentes. O entendimento dos mecanismos de geração e transmissão de calor leva ao rápido desenvolvimento das máquinas térmicas que possibilitaram a Revolução Industrial do século XIX. A surpreendente fenomenologia elétrica e magnética, quando estudada e dominada, abriu o caminho para a até então inimaginada expansão industrial do século XX. E a compreensão da matéria na sua intimidade quântica levou à extraordinária revolução eletrônica, das comunicações e da informática que marcaram de forma tão profunda as transformações da segunda metade do século XX. De forma similar, na medicina e na saúde, o conhecimento da anatomia e fisiologia humanas, associado ao avanço na compreensão da química de produtos naturais e sintética, promoveu avanços consideráveis, mais que duplicando a expectativa média de vida da espécie humana.

Ciência e inovação são, portanto, as duas faces de uma mesma moeda e constituem a força propulsora maior do avanço da humanidade ao longo de toda a sua história.

\section{A CIÊNCIA BRASILEIRA E SEU CENÁRIO ATUAL}

Após o descobrimento, fruto direto do grande centro de conhecimento e tecnologia que foi a Escola de Sagres, nossa história como nação foi marcada por três séculos iniciais de extrativismo colonial, em que a educação e o desenvolvimento foram fortemente inibidos e mesmo duramente reprimidos. De acordo com Capozzoli (2009), somente com a vinda da família real, no início do século XIX, abrem-se as portas da nação para edu-
GLAUCIUS OLIVA

é professor titular do Instituto de Física de São Carlos-USP e presidente do CNPq.

\section{FELIZARDO}

PENALVA DA SILVA

é professor

aposentado de

Genética da UnB

e chefe de

gabinete do CNPq. 
cadores, cientistas, técnicos, engenheiros e médicos, que puderam então se engajar no tardio, mas necessário, esforço desenvolvimentista do Brasil. As pioneiras faculdades de medicina da Bahia e do Rio de Janeiro, bem como as escolas de direito de Olinda e São Paulo, criam o ambiente de ensino necessário para a formação das lideranças transformadoras do país. A economia nacional fortemente dominada pela agricultura gera a demanda para a criação de diversas escolas agrícolas, como a Escola Agrícola da Bahia, a Escola Superior de Agronomia Luiz de Queiroz e outras similares em Pelotas, Rio de Janeiro, Lavras e Viçosa, que sustentaram a produção nacional subsequente ao abominável período da escravidão.

Em função dos graves problemas sanitários nos centros urbanos emergentes, são criados os primeiros núcleos de pesquisa médica, como os institutos Manguinhos e Vital Brazil, que promovem grande impacto na saúde pública nacional. É nesse cenário que, estimuladas pela forte influência cultural europeia, surgem as primeiras universidades, consolidadas como tal essencialmente em dois centros principais, a Universidade do Brasil, no Rio de Janeiro, e a Universidade de São Paulo, na capital paulista, a qual apenas em 1934 é estabelecida com a visão de investir na ciência e na cultura como forma de recuperar o prestígio e o poder político perdidos nos conflitos civis de 1930 e 1932 , como gravado emblematicamente em seu brasão: "Scientia Vinces".

Observa-se, portanto, no Brasil, o mesmo ciclo virtuoso de promoção e institucionalização do ensino e da pesquisa acoplado às demandas e necessidades do país. O desenvolvimento nacional sempre foi indelevelmente associado à incorporação de conhecimento como elemento fundamental na competitividade produtiva. Por um longo período de nossa história, o conhecimento e a tecnologia foram gerados fora do país, e somente incorporados mediante três processos básicos: a) a atração para o país de especialistas estrangeiros, que traziam consigo o conhecimento já adquirido e aqui o aplicavam; b) o envio dos filhos da pequena elite brasileira para estudar nas melhores escolas europeias, que foi o principal mecanismo de formação de quadros e lideranças para o país; c) a aquisição de máquinas e equipamentos importados, que serviram tanto à mecanização da agricultura como à incipiente industrialização da primeira metade do século XX.

Foi somente após a Segunda Guerra Mundial que o Brasil acordou definitivamente para a necessidade de investir em ciência e tecnologia. O maior conflito mundial só havia sido decidido pelo rápido e emergencial avanço do conhecimento e da tecnologia, como o alcançado na construção de aeronaves, navios de guerra e submarinos, nos sistemas de telecomunicações, na produção industrial de antibióticos, e no tão decisivo quanto trágico desenvolvimento e detonação das bombas nucleares que se abateram sobre Hiroshima e Nagasaki. As lideranças militares e civis brasileiras imediatamente compreenderam que nenhuma nação poderia garantir a soberania nacional e o desenvolvimento econômico e social se não fosse capaz de deter o conhecimento e a tecnologia necessários para tanto. É nesse período que são criadas a Sociedade Brasileira para o Progresso da Ciência, a Comissão Nacional de Energia Nuclear, o Centro Brasileiro de Pesquisas Físicas, a Coordenação de Aperfeiçoamento de Pessoal para o Ensino Superior (Capes) e o Conselho Nacional de Pesquisas (CNPq), hoje Conselho Nacional de Desenvolvimento Científico e Tecnológico. O desafio era tremendo, pois se tratava de um país com séculos de atraso na prática científica e enorme passivo educacional, tendo $85 \%$ de sua população no meio rural e com pouquíssimo acesso à educação.

Pouco mais de meio século nos separa desse período de mutação, e observamos hoje um país completamente distinto, nos aspectos não apenas científico e educacional, mas também econômico, produtivo, social e cultural. Temos atualmente uma comunidade científica e tecnológica pujante, expressa nos números da Plataforma Lattes do CNPq, onde se encontram hoje registrados perto de 2,5 milhões de currículos, entre os quais cerca de $500 \mathrm{mil}$ 
com mestrado ou doutorado, organizados nos mais de 27.500 grupos de pesquisa cadastrados no Censo 2010 do Diretório de Grupos de Pesquisa (DGP). O Brasil produz hoje 2,7\% de toda a ciência mundial e tem liderança reconhecida em várias áreas do conhecimento, como agricultura tropical, geofísica e engenharia associada à prospecção de petróleo e gás em águas profundas, e parasitologia, apenas para ficar em alguns exemplos. O Censo 2010 do sistema de ensino superior do Brasil, realizado pelo Inep, contabiliza no país 2.377 instituições de ensino superior, das quais 253 são universidades, que receberam naquele ano 6,4 milhões de matrículas e formam anualmente mais de um milhão de graduados. Em 2010 a pós-graduação brasileira já contava com 2.436 cursos de mestrado acadêmico, 243 mestrados profissionais e 1.420 de doutorado, regularmente avaliados e certificados pela Capes, os quais têm cerca de $180 \mathrm{mil}$ estudantes e que já formam cerca de $40 \mathrm{mil}$ mestres e 12 mil doutores por ano. E todo esse esforço tem resultado em extraordinário avanço para o país, como veremos a seguir.

\section{IMPACTOS ECONÔMICOS E SOCIAIS DA CIÊNCIA BRASILEIRA}

Tomando como exemplos os maiores setores da economia, como agronegócio, óleo e gás, mineração, energia, aeroespacial, construção civil e indústrias de base e de transformação, em todos os casos vemos que a universidade e os institutos de pesquisa brasileiros têm tido papel essencial, seja na formação de pessoal qualificado com a habilidade e competências necessárias para conduzir os avanços alcançados, seja no desenvolvimento tecnológico sem o qual a profunda transformação do país não teria ocorrido da forma como se observa hoje. Foi no seio das melhores escolas agrícolas deste país que se formaram os pesquisadores doutores que permitiram à Embrapa promover o salto produtivo de nosso agronegócio, espalhan- do por todo o país culturas antes limitadas a climas e solos restritos a poucas regiões.

É importante lembrar que meio século atrás o Brasil ainda importava alimentos e ainda recebia doações regulares de leite em pó de organismos internacionais de combate à fome, como o Usaid, e hoje se constitui em um dos maiores exportadores de alimentos para o mundo. Até cerca de trinta anos atrás a produção de petróleo nacional era restrita a poços continentais. Foi quando a Petrobras decidiu investir fortemente em pesquisa e desenvolvimento, criando o seu centro de pesquisas (Cenpes) dentro do campus de uma grande universidade brasileira (UFRJ), que abriga um dos melhores programas de pós-graduação em engenharia do país (Coppe). Essa decisão estratégica levou a companhia a se tornar líder mundial na exploração de petróleo em águas profundas e a ocupar o terceiro lugar entre as maiores empresas petrolíferas de todo o planeta. A decisão estratégica da Força Aérea Brasileira de estabelecer um polo de conhecimento e desenvolvimento aeronáutico iniciou-se com a criação do Instituto Tecnológico da Aeronáutica, o qual promoveu a formação dos recursos humanos de excelência na área, o que propiciou ao Brasil ter hoje a terceira maior empresa mundial de aviação civil e militar (Embraer).

A engenharia nacional, consolidada nas pesquisas e na formação de recursos humanos em grandes escolas, algumas delas centenárias, permitiu a projeção e a construção de grandes empreendimentos civis, como usinas hidrelétricas e uma extensa malha rodoviária nacional, bem como a rápida industrialização do último meio século. Da mesma forma, a saúde do país beneficiou-se largamente da formação de recursos humanos e das pesquisas realizadas nas nossas melhores escolas médicas e correlatas, que praticamente duplicaram a expectativa de vida ao nascer do cidadão brasileiro desde a Segunda Guerra Mundial. Do ponto de vista educacional e social, o país também é outro, pois com os professores formados nas instituições de ensino superior brasileiras viabilizou-se o grande esforço nacional de universalização 
do acesso à educação básica, embora enfrentemos ainda grandes desafios no que diz respeito à qualidade do ensino ofertado.

Não é, portanto, nenhum exagero afirmar que essencialmente tudo que tem dado certo no desenvolvimento nacional tem de alguma forma a participação da ciência e da educação superior brasileiras.

\section{CIÊNCIA E INOVAÇÃO COMO PLATAFORMA ESSENCIAL PARA O PLENO DESENVOLVIMENTO SUSTENTÁVEL}

Os cenários macroeconômico, social e ambiental, tanto nacional como mundial, apontam inexoravelmente para a inovação e o desenvolvimento científico e tecnológico como o grande eixo da retomada do crescimento econômico e social dos países e do planeta. Países tradicionais em ciência e tecnologia, como os Estados Unidos, as principais economias da Europa e o Japão, embora enfrentando os graves efeitos da crise econômica e financeira sistêmica, têm apostado decididamente nos investimentos em ciência, tecnologia e inovação, com estímulos tanto à busca pelas fronteiras do conhecimento como a sua apropriação pelas empresas e pela sociedade. Somente no período de deflagração da crise econômica internacional de 2008-09, o governo americano anunciou simultaneamente fortes cortes nos gastos públicos, porém preservando um crescimento global de mais de 100 bilhões de dólares nos investimentos em pesquisa e desenvolvimento em áreas estratégicas portadoras de futuro. Da mesma forma, países emergentes como China, Coreia do Sul, Índia e tantos outros têm se destacado em seu esforço desenvolvimentista exitoso pelos fortes investimentos em educação, ciência e tecnologia.

O exemplo coreano é emblemático, pois no início dos anos 80 apresentava indicadores econômicos, sociais e educacionais semelhantes aos do Brasil e, em meio à grave crise econômica deflagrada pelo contexto internacional no início dos anos 80 , decide cortar em muitos setores, porém investir muito mais em ciência e tecnologia, tanto nos meios acadêmicos como empresariais. Em menos de trinta anos, esse país investe 3,5\% de seu PIB em pesquisa e desenvolvimento e, paralelamente, já alcançou níveis econômicos e de desenvolvimento humano (PIB per capita) comparáveis aos dos países centrais.

A China tem igualmente dado exemplos do sucesso na estratégia de investimentos firmes e vultosos tanto na formação própria como na atração de recursos humanos de excelência nas áreas estratégicas para o desenvolvimento do país, na consolidação de algumas de suas melhores instituições acadêmicas como universidades de classe mundial, com expressiva produção científica, e no estímulo à inovação industrial por meio de uma rede de institutos de pesquisa tecnológica.

$\mathrm{O}$ aspecto talvez mais importante desse processo de escala mundial tem sido a crescente preocupação em acoplar o desenvolvimento amplo do planeta à sustentabilidade ambiental, com fortes investimentos em energias limpas, economia verde, redução de emissões de gases de efeito estufa e atenção às mudanças climáticas globais. $\mathrm{O}$ próximo ciclo virtuoso de crescimento econômico mundial será centrado em ciência, tecnologia e inovação para o desenvolvimento sustentável. Como país detentor da maior biodiversidade do planeta, vastos recursos aquíferos e minerais, grande área de terras agricultáveis, florestas e mar territorial de dimensões continentais, e pelos avanços científicos tecnológicos já alcançados, o Brasil tem oportunidade singular e estratégica de liderar o crescimento da economia verde mundial.

\section{SEM CIÊNCIA DE QUALIDADE E EM QUANTIDADE, NÃO HÁ INOVAÇÃO POSSÍVEL}

Nenhum país do mundo conseguiu alcançar o pleno desenvolvimento da nova economia do conhecimento e da inovação sem uma 


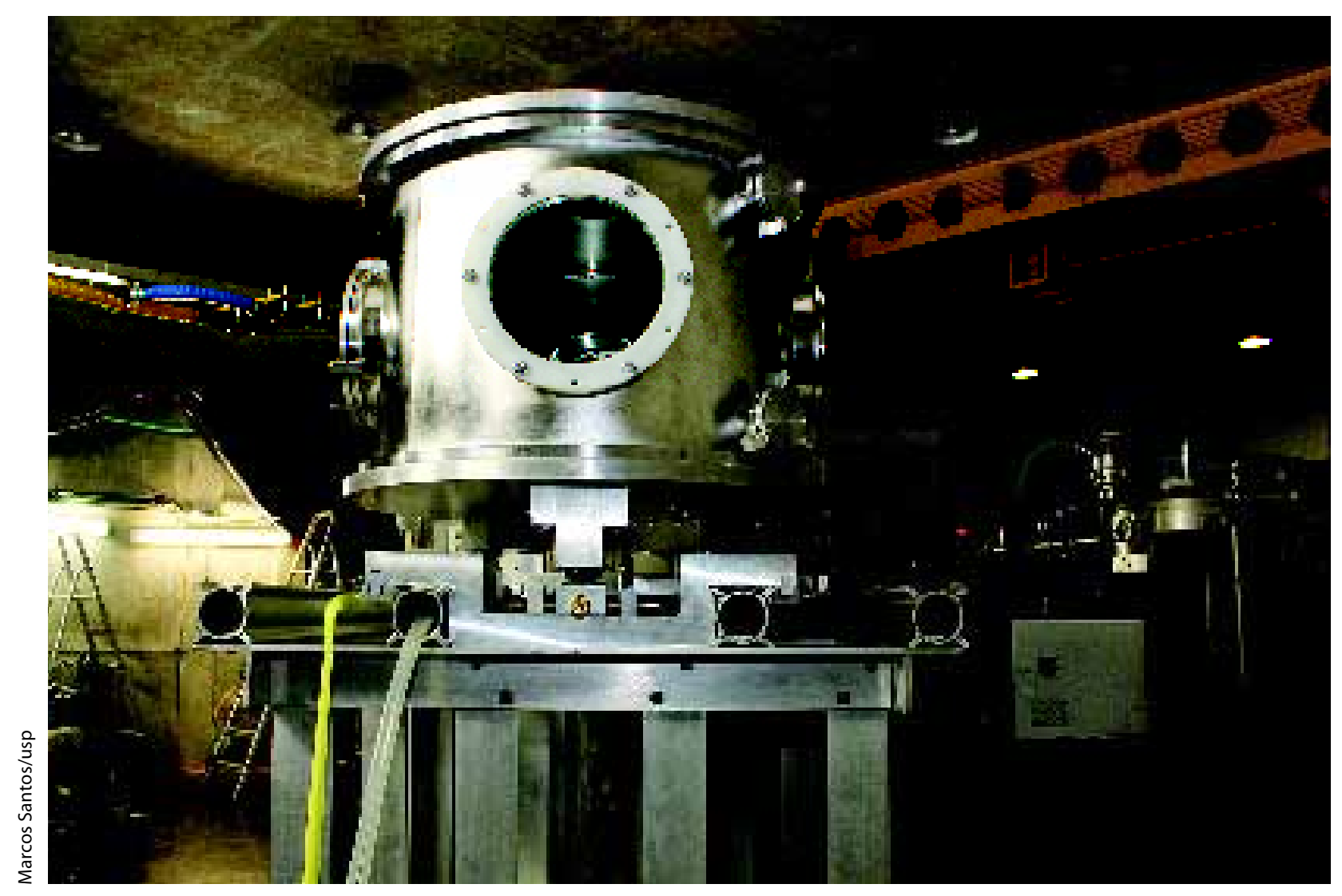

forte inserção em ciência básica de qualidade e em quantidade compatível com as necessidades nacionais. Nesse sentido, a ciência brasileira tem feito sua parte. São bem conhecidos os exemplos de sucesso já alcançados tanto na área tecnológica, com importantes benefícios políticos, econômicos e sociais, quanto na científica em que, além do seu valor intrínseco, fornecem o conhecimento acumulado resultante da curiosidade para se ampliarem as respostas aos desafios da necessidade. Na verdade, com ritmos distintos, ciência e inovação cresceram significantemente nos últimos anos e esse crescimento resultou de uma política específica dirigida para se alcançar tal resultado. Cresceram e se diferenciaram como produto natural resultante dos programas de formação de recursos humanos e financiamento à pesquisa adotados no Brasil. Consequentemente, é de esperar que eles continuem crescendo e se diferenciando, com recursos, planejamento e gestão que promovam o avanço do conhecimento e do desenvolvimento humano, eco- nômico, social e ambiental almejados pela nação brasileira. Por outro lado, é fundamental identificar todas as variáveis envolvidas para alterar algumas delas na tentativa de amplificar a qualidade da produção científica, tecnológica ou mesmo da inovação. A equação em jogo é a dependência da tecnologia e inovação em relação à disponibilidade e à qualidade do conhecimento disponível.

Assim sendo, um fator determinante que não pode ser olvidado é o dos critérios e indicadores utilizados para analisar os aspectos quantitativos e qualitativos da produção, tanto científica quanto tecnológica. O julgamento pelos pares, que consideram o número de trabalhos publicados em revistas indexadas, citações e envolvimento na formação de recursos humanos, tem sido aceito, mesmo se eventualmente questionado, como um padrão de análise da produção do conhecimento. Sua adoção extensiva foi essencial para a promoção do expressivo avanço da ciência nacional nos últimos trinta anos, porém esse modelo de avaliação não apresenta o estímulo e a va-
Acelerador de partículas do Instituto de Física da USP 
lorização necessários ao maior engajamento dos cientistas brasileiros na inovação e na divulgação da ciência para a sociedade. $\mathrm{Na}$ análise da produção tecnológica, a situação é mais delicada porque os indicadores mais frequentes, como o registro de patentes e o impacto econômico, não chegam a compor um padrão pacífico e de fácil aferição. Muitas patentes visam apenas à proteção da produção intelectual, sem qualquer perspectiva de vir a resultar em desenvolvimento, produto ou processo. Além disso, a patente é um indicador de longo prazo porque não se sabe quando sairá do papel para a realidade. Quanto ao impacto econômico, não podemos aferi-lo com segurança enquanto não for inserido no sistema de produção.

Outros itens de produção em inovação devem ser também adotados, como a realização de projetos de pesquisa e desenvolvimento em parceria com empresas ou órgãos públicos; as atividades de assessoria e consultoria que promovam a incorporação de conhecimento a produtos, processos ou serviços; a produção de softwares com ou sem registro; a extensão tecnológica e social; e a formação de mestres e doutores para a indústria e a sociedade, entre outros.

Em relação ao conhecimento produzido, a sua avaliação é mais pacífica e conhecida, o que dispensa maiores considerações. Alguns aspectos, entretanto, são dignos de nota. De acordo com Zago (2011), um dado estimulante resultante das análises da base de publicações científicas indexadas, Web of Science, é o das parcerias internacionais. Há uma correlação positiva e significativa entre os trabalhos resultantes de parceria internacional e o número de citações. Admitindo-se que esse é um indicador de qualidade, mesmo com ressalvas e restrições, esse tipo de informação é fundamental para se avaliar a produção científica do Brasil e compará-la com a de outros países. Essa comparação nos permite observar que, nos últimos anos, a produção brasileira só cresceu menos que a da China, e que a produção total atual é maior que a de países desenvolvidos como a Suíça e a Suécia. Detentor de cerca de 3\% da produ- ção científica mundial e em curva ascendente, o Brasil não pode ter a produção de conhecimento acusada como responsável pelo atraso na produção tecnológica e inovação.

Ainda no campo das comparações, é qualitativo, porém válido, observar que a área com maior produção de conhecimento pode influir no maior avanço tecnológico, reforçando o princípio de que o avanço em tecnologia e inovação depende de uma produção de conhecimento diferenciada. De acordo com aquelas informações, por exemplo, enquanto nós produzimos mais e aparentemente melhor conhecimento nas ciências da vida (biologia, medicina, agronomia), com forte impacto no nosso agronegócio, na China os primeiros lugares são ocupados pelas ciências exatas (física, engenharia e materiais), que resultam no seu forte desenvolvimento e competitividade industrial. Além disso, no Brasil os pesquisadores profissionais são significativamente mais numerosos nas universidades do que nas empresas. Talvez residam aí as explicações para a menor produtividade brasileira na área tecnológica quando comparada com a da China e de outras economias concorrentes.

Admitindo como premissa verdadeira a afirmação de que a tecnologia e a inovação só acontecem respaldadas em uma produção de conhecimento qualitativa e quantitativamente diferenciadas, os dados disponíveis sugerem que o Brasil tem alcançado produção compatível, a qual deve ser sustentada de forma decidida, bem como adicionada do estímulo ao enfrentamento dos desafios e prioridades da nossa realidade.

\section{OS DESAFIOS DA CIÊNCIA BRASILEIRA PARA A PRÓXIMA DÉCADA}

A análise retrospectiva da evolução da ciência no Brasil deixa claro que foi adotado um modelo próprio de formação de recursos humanos e financiamento à pesquisa apoiado na proposta de estruturação e consolidação de 
uma pós-graduação acadêmica, e que esse modelo vem apresentando resultados satisfatórios em relação à produção de conhecimento.

A pouca atenção dada à área de tecnologia e inovação, nesse processo evolutivo, talvez seja a melhor explicação para a situação atual de baixa produtividade nesse setor, de acordo com os indicadores adotados. Além disso, é possível identificar também outros fatores que podem ser responsabilizados pela situação atual. Sem pretender ser exaustivo, eles podem ser sumarizados como se segue.

Em primeiro lugar, pelo baixo envolvimento do setor empresarial no esforço para desenvolver tecnologia e inovação. Apesar dos progressos já alcançados, a grande preocupação do setor diz respeito ao denominado “custo Brasil", conforme Galembeck (2011). Nesse caso específico, representado particularmente pelas cargas trabalhista e tributária, dificultando a geração de empregos e influindo negativamente na implantação de núcleos de pesquisas. Ainda nesse âmbito, compõem a pauta os modestos incentivos fiscais e uma legislação pouco amigável. A inovação ocorre particularmente no ambiente de produção da empresa e, pelo menos na fase de desenvolvimento, esses incentivos e correções podem e devem ser considerados.

Em segundo lugar, pelo perfil da pós-graduação brasileira, que ainda hoje atribui pouco valor ou estímulo à formação tecnológica e consequentemente não promove a absorção do pessoal qualificado no ambiente industrial. No profundo estudo do perfil dos egressos da pós-graduação no Brasil realizado pelo Centro de Gestão e Estudos Estratégicos (2011), no universo dos doutores formados entre 1996 e 2006, 77\% estavam empregados em 2008 no setor de educação e outros $11 \%$ no governo. Outro componente é que somente recentemente vem sendo reforçada a preocupação com a formação de mais engenheiros, e aqueles que chegam a cursar pós-graduação raramente são absorvidos pela indústria. O Programa Ciência sem Fronteiras, do governo federal, busca justamente transformar esse quadro, oferecendo a pelo menos 100 mil jo- vens estudantes e pesquisadores brasileiros a oportunidade de realizar estágios e pesquisas em ambientes educacionais e profissionais em que a inovação, o empreendedorismo e a competitividade já são o padrão.

Ainda no âmbito retrospectivo, há que se rever e complementar a legislação, particularmente para diminuir a carga burocrática que envolve o sistema devido aos "vácuos legais". Por exemplo, aplicar a lei de licitações na utilização de recursos subsidiados ou concedidos a fundo perdido para o desenvolvimento de projetos é ato questionável, mesmo tendo uma base legal. Essa lei surgiu para regulamentar a concorrência pública para contratos de prestação de serviços e aquisição de bens e insumos, não para regulamentar a utilização dos magros recursos destinados a projetos de pesquisas.

Um terceiro aspecto da análise retrospectiva é o dos exemplos que deram certo. Agronegócios, energia (petróleo, gás e bioetanol), aviação, computação (bancária e eleitoral) e outros são todos exemplos baseados em um mesmo modelo: identificação de uma prioridade, capacitação de pesquisadores e investimento focado em áreas e grupos consistentes. A identificação das prioridades já foi devidamente explorada e elas estão explicitadas no documento denominado "Estratégia Nacional para Ciência, Tecnologia e Inovação - ENCTI - 2011-2015" (CGEE, 2011).

Entramos assim no campo prospectivo, onde fica evidente que se utiliza o mesmo modelo, com algumas correções de rumo que permitem adotar novas estratégias. O referido documento ENCTI resultou de um grande esforço do governo para estabelecer diretrizes e traz claramente definidas, ao lado dos desafios, as prioridades estabelecidas como referenciais que o CNPq ajudou a construir.

O documento ENCTI-2011-2015 aponta como desafios:

a) redução da defasagem científico-tecnológica que nos separa dos países mais avançados do ponto de vista científico, tecnológico e de inovação. Como vimos, apesar do aumento significativo da produção nacional aferida 
pelos indicadores convencionais ainda estamos longe de alcançar os países líderes sob os aspectos da relevância e impacto;

b) expansão e consolidação da liderança brasileira na economia do conhecimento natural. Esse desafio nos coloca numa situação privilegiada, pois deve ser enfrentado tendo como fundamento a riqueza dos nossos bens naturais;

c) ampliação da base para sustentabilidade ambiental e desenvolvimento de uma economia de baixo carbono. A sustentabilidade busca o equilíbrio entre preservação e desenvolvimento que alcance e beneficie toda a sociedade;

d) melhoria na inserção internacional. Além do estímulo à parceria e multidisciplinaridade, que são exigências da ciência moderna, esse esforço pode representar controle de qualidade e maior chance de sucesso no desenvolvimento de projetos;

e) superação da pobreza e redução das desigualdades sociais e regionais. Esse é um desafio que ultrapassa o âmbito de CT\&I e alcança todos os setores da sociedade.

O documento relaciona também os programas prioritários e a inovação emerge como uma estratégia natural de ação que pode contribuir para a superação desses desafios.

Iniciamos este artigo fazendo referência a Darwin e concluímos com ele ao constatarmos que a identificação de desafios e a seleção de prioridades respaldadas em uma produção de conhecimento diferenciada podem constituir o caminho natural equilibrado para se vencer a corrida de longa distância e alcançar o desenvolvimento científico, tecnológico e a inovação comprometido com o desenvolvimento social sustentável.

\section{BIBLIOGRAFIA}

CAPOZZOLI, Ulisses (ed. e org.). "História da Ciência no Brasil”, in Scient. American, v. 1, São Paulo, Dueto, 2009.

CGEE. Estratégia Nacional de Ciência, Tecnologia e Inovação - 2011/2015. MCT, 2011

GALEMBECK, F. "Avaliação do Perfil Atual da Pesquisa Aplicada no Brasil", in Ricardo U. Sennes e A. Britto Filho (orgs.). Inovações Tecnológicas no Brasil. São Paulo, Cultura, 2011.

ZAGO, M. A. "Evolução e Perfil da Produção Científica Brasileira", in Ricardo U. Sennes e A. Britto Filho (orgs.). Inovações Tecnológicas no Brasil. São Paulo, Cultura, 2011. 